\title{
Estimación de parámetros genéticos para características de crecimiento en borregos Katahdin usando diferentes modelos
}

\section{Genetic parameter estimates for growth traits in Katahdin sheep using different models}

\author{
Coralia Inés V. Manzanilla Pecha, José Antonio Torres Vázquez ${ }^{\mathrm{b}}$, Aurelio Borrayo Zepedac, \\ Ángel Ríos Utrera ${ }^{d}$, Juan José Baeza Rodríguez ${ }^{\mathrm{a}}$, Guillermo Martínez Velázquez ${ }^{\mathrm{c}}$, Vicente \\ Eliezer Vega Murillo ${ }^{\mathrm{d}}$, Moisés Montaño Bermúdez ${ }^{\mathrm{b}}$
}

\begin{abstract}
RESUMEN
Se estimaron parámetros genéticos para características de crecimiento en corderos Katahdin, usando seis variantes del modelo animal. Se usó información de pesos al nacimiento $(B W ; n=13,099)$, al destete ajustado a 75 d $(W W ; n=11,509)$ y posdestete ajustado a 120 d (AW; $n=6,886)$ tomada durante 7 años (2004-2010) en 20 estados de la República Mexicana. Los análisis se hicieron ignorando o incluyendo efectos maternos. El modelo más sencillo incluyó el efecto genético aditivo directo como el único efecto aleatorio. El modelo más completo incluyó los efectos genéticos directo y materno, la covarianza entre ellos, y el efecto del ambiente permanente materno. Para seleccionar el mejor modelo se usó la prueba de razón de verosimilitud. Cuando los efectos maternos no fueron incluidos en el modelo, los estimadores de la heredabilidad directa y de la varianza genética directa resultaron sobreestimados. Las heredabilidades directas con el mejor modelo fueron $0.18 \pm 0.03,0.30 \pm 0.04$ y $0.20 \pm 0.05$ para $\mathrm{BW}, \mathrm{WW}$ y AW, respectivamente. Las heredabilidades maternas también variaron dependiendo del modelo, de 0.05 a $0.23,0.00$ a 0.12 , y 0.09 a 0.25 para $\mathrm{BW}$, WW y AW. El ignorar los efectos maternos en el modelo resultaría en una evaluación genética equivocada para las características de crecimiento en borregos Katahdin.
\end{abstract}

Palabras ClaVe: Peso al nacer, Peso al destete, Katahdin, Parámetros genéticos.

\begin{abstract}
Genetic parameters for growth traits of Katahdin lambs were estimated using six variants of the animal model. Data on birth weight $(\mathrm{BW} ; \mathrm{n}=13,099)$, weaning weight adjusted to $75 \mathrm{~d}(\mathrm{WW} ; \mathrm{n}=11,509)$ and postweaning weight adjusted to $120 \mathrm{~d}(\mathrm{AW} ; \mathrm{n}=6,886)$ were collected for seven years (2004-2010) in 20 states across Mexico. Analyses were carried out by ignoring or including maternal effects. The simplest model included the direct additive genetic effect as the only random effect. The most complete model included direct and maternal genetic effects, their covariance, and the maternal permanent environmental effect. Selection of the best model was based on likelihood-ratio test. When maternal effects were not taken into account, estimates of direct heritability and direct genetic variance were overestimated for all traits. Direct heritability estimates for the best model were $0.18 \pm 0.03,0.30 \pm 0.04$, and 0.20 \pm 0.05 for BW, WW and AW, respectively. Maternal heritability estimates also varied depending on the model; estimates ranged from 0.05 to $0.23,0.00$ to 0.12 , and 0.09 to 0.25 for $\mathrm{BW}$, WW and $\mathrm{AW}$. Ignoring maternal effects in the model would result in inaccurate genetic evaluation for growth traits in Katahdin sheep.
\end{abstract}

KEY WORDS: Birth weight, Weaning weight, Katahdin, Genetic parameters.

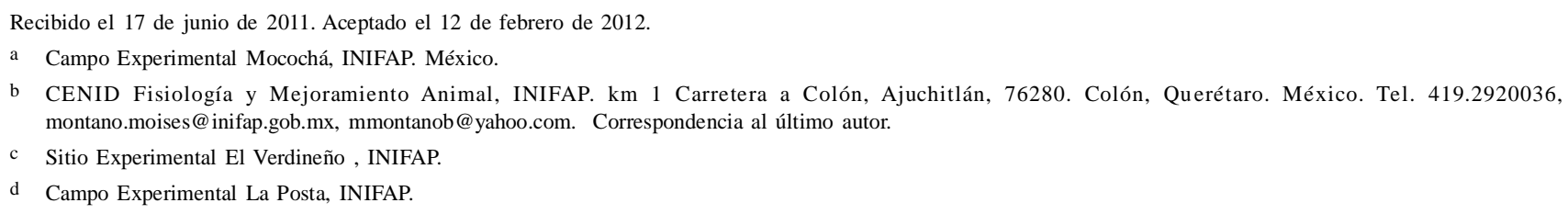




\section{INTRODUCCION}

Se puede esperar que los efectos maternos sean más importantes en borregos que en ganado bovino o porcino, debido a una mayor variación en el tamaño de la camada en borregos y al hecho de que muchos corderos dependen parcialmente de la producción de leche de la madre hasta un peso

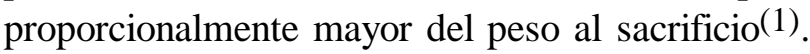
Katahdin es la raza de borregos con más animales registrados en México; de acuerdo con Wildeus(2), esta raza compuesta ha sido seleccionada para crecimiento, conformación, prolificidad y en contra de la presencia de lana. En México, los corderos se comercializan para consumo, normalmente después del destete; de esta forma, los pesos al nacimiento y al destete son características de importancia económica por su efecto a través del crecimiento predestete. Hay muchos factores que afectan el peso al nacimiento y la ganancia predestete en corderos; entre ellos se incluyen los efectos genéticos directos y maternos, así como los ambientales(3). En borregos, algunos estudios (4-7) han encontrado que los efectos del ambiente materno tienen una contribución importante en la varianza fenotípica. La incorporación de los efectos maternos ambientales en el modelo de análisis contribuye a la precisión de los estimadores de los parámetros y los valores genéticos, mientras que la exclusión puede llevar a estimadores sesgados. Los parámetros genéticos pueden variar debido a raza, ubicación y rebaño ${ }^{(8)}$; por ello, es importante contar con estimadores apropiados de los parámetros genéticos, para diseñar estrategias de selección adecuadas y para estimar valores genéticos adecuados. Sin embargo, en la raza Katahdin se conoce poco de la variación genética para características de crecimiento predestete y de otros componentes de la efíciencia económica de la producción.

Hay muchos informes de estimadores de parámetros genéticos para características de crecimiento en muchas razas de borregos $(9-13)$. Algunos estudios han encontrado una correlación negativa entre efectos aditivos directos y maternos para pesos al

\section{INTRODUCTION}

Maternal effects may be expected to be more important in sheep than in cattle or swine because of the greater relative variation in litter size in sheep and the fact that many lambs are partially dependent on their mother's milk supply until they have achieved a higher proportion of their slaughter weight ${ }^{(1)}$. Katahdin is the breed of sheep with more registered animals in Mexico. According to Wildeus ${ }^{(2)}$, this composite breed has been selected for growth rate, mutton conformation, prolificacy, and against wool. In Mexico, lambs are normally marketed after weaning for consumption; thus, birth and weaning weight are traits of potential economic importance through their effects on preweaning growth rate. Many factors affect birth weight and preweaning growth of lambs. These factors include direct genetic, maternal genetic and environmental effects $(3)$. In sheep, numerous studies $(4,5,6,7)$ have indicated that maternal environmental effects have sizeable contributions to the overall phenotypic variance. Incorporation of maternal environmental effects in the analytical model will thus contribute to the accuracy of estimates of genetic parameters and breeding values, while exclusion may lead to biased estimates. Genetic parameters may vary because of breed, location and flock ${ }^{(8)}$ hence, appropriate parameter estimates for growth traits are important for adequate breeding strategies and for accurate breeding value estimation. However, little is known about genetic variation within the Katahdin breed for preweaning growth traits and other components of the economic efficiency of sheep production.

Estimates of genetic parameters for growth traits of many breeds of sheep have been extensively reported $(9,10,11,12,13)$. Numerous studies have found a negative correlation between additive direct and additive maternal effects for birth and weaning weights of various sheep breeds $(3,8)$. Positive relationships have also been reported ${ }^{(8)}$. However, there are no results on maternal effects and correlation between additive direct and additive maternal effects for birth and weaning weights of 
nacimiento y destete en varias razas $(3,8)$. También se han encontrado correlaciones positivas ${ }^{(8)}$. Sin embargo, no hay información de efectos maternos y correlación entre efectos aditivos directos y maternos para pesos al nacer y destete en corderos Katahdin. De esta manera, la información obtenida será útil para el diseño de programas de mejoramiento en borregos Katahdin en México. El objetivo del presente estudio fue estimar componentes de (co)varianza y parámetros genéticos para peso al nacer, peso al destete ajustado a $75 \mathrm{~d}$, y peso posdestete ajustado a $120 \mathrm{~d}$ en borregos Katahdin usando modelos que ignoran o incluyen efectos maternos.

\section{MATERIALES Y METODOS}

La información productiva y de pedigrí se obtuvo de la Asociación Mexicana de Criadores de Ovinos (AMCO) de animales nacidos entre 2004 y 2010. Se obtuvo información de pesos al nacer, destete y posdestete de 131 rebaños en 20 estados de México. Después de la edición de la información, el archivo final contenía 13,099 registros de peso al nacer (BW), 11,509 de pesos al destete (WW), y 6,886 de pesos posdestete (AW). El peso al nacer fue de corderos nacidos vivos. Los pesos al destete y posdestete se ajustaron a 75 y 120 días de edad, respectivamente. Los corderos fueron hijos de 618
Katahdin lambs. Thus, the information generated would be useful in designing improvement programs for Katahdin sheep in Mexico. The main objective of the present study was to estimate (co)variance components and genetic parameters for birth weight, weaning weight adjusted to $75 \mathrm{~d}$, and postweaning weight adjusted to $120 \mathrm{~d}$ in Katahdin sheep with models that ignore or include maternal effects.

\section{MATERIALS AND METHODS}

Data and pedigree information were provided by Mexican Association of Sheep Breeders (AMCO) for the period 2004-2010. Birth, weaning and postweaning weight records from 131 flocks in 20 states across Mexico were collected. After edition of the records, the final data set comprised 13,099 records for birth weight (BW), 11,509 for weaning weight (WW), and 6,886 for postweaning weight (AW). Birth weight was from lambs that were born alive. Individual weaning and postweaning weight records were adjusted to 75 and $120 \mathrm{~d}$ of age, respectively. The lambs were the progeny of 618 rams and 5,820 ewes. The summary statistics and characteristics of the data structure are shown in Table 1.

(Co)variance components and genetic parameters were estimated using multi-trait derivative-free

Cuadro 1. Descripción estadística y estructura de la información para características de crecimiento de corderos Katahdin

Table 1. Summary statistics and structure of the data for growth traits of Katahdin lambs

\begin{tabular}{lccc}
\hline & Birth weight & Weaning weight & $120 \mathrm{~d}$ weight \\
\hline Mean, kg & 3.7624 & 21.7358 & 26.1509 \\
Maximum, kg & 1.500 & 8.8330 & 10.032 \\
Minimum, kg & 6.900 & 39.864 & 44.739 \\
Standard deviation, kg & 0.9188 & 4.4961 & 5.1253 \\
Coefficient of variation, \% & 24.42 & 20.69 & 19.60 \\
Number of records & 13,099 & 11,509 & 6,886 \\
Number of animals in the pedigree & 18,367 & 18,367 & 10,711 \\
Contemporary groups & 677 & 667 & 583 \\
Number of dams & 5,820 & 5,820 & 3701 \\
Number of sires & 618 & 618 & 426 \\
\hline
\end{tabular}


carneros y 5,820 borregas. El resumen de la información y la estructura de la misma se muestran en el Cuadro 1.

Los componentes de (co)varianza y los parámetros genéticos se estimaron con el programa de máxima verosimilitud restringida libre de derivadas para características múltiples (MTDFREML), con un modelo animal para una sola característica y usando seis modelos estadísticos. En todos los modelos se consideró el grupo contemporáneo como efecto fijo. Los grupos contemporáneos (CG) se formaron agrupando animales nacidos o destetados en el mismo año, estación y rebaño. Los grupos contemporáneos con menos de cinco observaciones se eliminaron del análisis, así como los corderos sin pesos al nacimiento. Tosh y $\operatorname{Kemp}^{(9)}$ demostraron que la varianza del error de predicción es menor cuando se excluyen los registros de animales de grupos contemporáneos con menos de cuatro individuos.

Las épocas fueron: 1) enero a marzo, 2) abril a junio, 3) julio a septiembre, y 4) octubre a diciembre. Otros efectos fijos fueron sexo, tipo de nacimiento (sencillo, doble, triple), y edad de la madre como covariable lineal en todas las características. La parte aleatoria varió en cada modelo. El modelo 1 incluyó solamente el efecto genético directo como efecto aleatorio; el 2 los efectos genético directo y el ambiental permanente materno; el 3 los efectos genéticos directo y materno, suponiendo que la covarianza entre ellos es igual a 0 ; el 4 los efectos genéticos directo y materno y el del ambiente permanente materno, suponiendo que la covarianza entre los efectos genéticos es igual a 0; el 5 los efectos genéticos directo y materno, suponiendo que la covarianza entre ellos es diferente de 0 , y el 6 los efectos genéticos directo y materno y el del ambiente permanente materno, suponiendo que la covarianza entre los efectos genéticos es diferente de 0 . Se consideró que la convergencia se alcanzó cuando la varianza de -2(logaritmo de la verosimilitud) en el simplex fue menor que 1 x $10^{-9}$. Una vez alcanzado el criterio de convergencia, el restricted maximum likelihood (MTDFREML) software, from single trait animal models, applying six statistical models. In all models contemporary group was used as a common fixed effect. Contemporary groups (CG) were formed by grouping animals born or weaned in the same year, season and flock. Contemporary groups with less than five observations were eliminated from the database as were individuals with missing data for birth weight. Tosh and Kemp ${ }^{(9)}$ demonstrated that the prediction error variance was minimized when records for animals in contemporary groups of four or less were excluded.

The seasons were: 1) January to March, 2) April to June, 3) July to September, and 4) October to December. Additional fixed effects were sex, type of birth (single, twin, triple), and age of dam as a linear covariable for all traits. The random part varied in each model. Model 1 included the direct genetic effect as the only random effect. Model 2 included direct genetic as well as maternal permanent environmental effects. Model 3 included direct and maternal genetic effects, assuming a covariance equal to zero between them. Model 4 included direct and maternal genetic effects, and maternal permanent environmental effects, assuming a covariance equal to zero between genetic effects. Model 5 included direct and maternal genetic effects, with a covariance between them different from zero, and Model 6 included the direct and maternal genetic effects, and the maternal permanent environmental effects, with covariance between genetic effects. Convergence was considered to have been reached when the variance of -2(logarithm of the likelihood) in the simplex was less than $1 \mathrm{x}$ $10^{-9}$. Once the interim convergence criterion was reached, ten restarts were continued to confirm global convergence.

Models in matrix notation were as follows:

Model 1: $\mathrm{Y}=\mathrm{Xb}+\mathrm{Z}_{\mathrm{a}} \mathrm{a}+\mathrm{e}$

Model 2: $Y=X b+Z_{a} a+Z_{c} c+e$

Model 3: $Y=X b+Z_{a} a+Z_{m} m+e \quad$ with $\operatorname{cov}(a, m)=0$

Model 4: $Y=X b+Z_{a} a+Z_{m} m+Z_{c} c+e$ with $\operatorname{cov}(a, m)=0$ 
procedimiento se reinició diez veces para confirmar que se alcanzó una convergencia global.

Los modelos en notación matricial fueron como sigue:

Modelo 1: $Y=X b+Z_{a} a+e$

Modelo 2: $Y=X b+Z_{a} a+Z_{c} c+e$

Modelo 3: $Y=X b+Z_{a} a+Z_{m} m+e \quad \operatorname{con} \operatorname{cov}(a, m)=0$

Modelo 4: $Y=X b+Z_{a} a+Z_{m} m+Z_{c} c+e \operatorname{con} \operatorname{cov}(a, m)=0$

Modelo 5: $Y=X b+Z_{a} a+Z_{m} m+e \quad \operatorname{con} \operatorname{cov}(a, m)=A \sigma_{a m}$

Modelo 6: $\mathrm{Y}=\mathbf{X b}+\mathrm{Z}_{\mathrm{a}} \mathrm{a}+\mathrm{Z}_{\mathrm{m}} \mathrm{m}+\mathrm{Z}_{\mathrm{c}} \mathrm{c}+\mathrm{e} \quad \operatorname{con} \operatorname{cov}(\mathrm{a}, \mathrm{m})=\mathbf{A} \sigma_{\mathrm{am}}$

Donde $\mathbf{Y}=$ vector de observaciones (BW, WW y $\mathrm{AW}) ; \mathbf{b}=$ vector de efectos fijos que incluye grupo contemporáneo, y tipo de nacimiento o destete; $\mathbf{a}=$
Model 5: $Y=X b+Z_{a} a+Z_{m} m+e \quad$ with $\operatorname{cov}(a, m)=A \sigma_{a m}$

Model 6: $Y=X b+Z_{a} a+Z_{m} m+Z_{c} c+e \quad$ with $\operatorname{cov}(a, m)=A \sigma_{a m}$

Where $\mathbf{Y}=$ vector of observations (BW, WW and $\mathrm{AW}) ; \mathbf{b}=$ fixed effects vector including contemporary group and type of birth or weaning; $\mathbf{a}=$ random direct additive genetic effects vector; $\mathbf{m}=$ random maternal genetic effects vector; $\mathbf{c}=$ random permanent environmental effects vector; $\mathbf{e}=$ random residual effects vector; $\mathbf{X}, \mathbf{Z}_{\mathbf{a}}, \mathbf{Z}_{\mathbf{m}}, \mathbf{Z}_{\mathbf{c}}=$ incidence matrices relating observations with fixed, direct genetic, maternal genetic and permanent environmental effects, respectively; $\mathbf{A}=$ numerator relationship matrix; $\sigma_{\mathrm{am}}=$ covariance between direct and maternal genetic effects.

Cuadro 2. Componentes de (co)varianza para modelos usados para características de crecimiento de borregos Katahdin

Table 2. (Co)variance components for models used in growth traits of Katahdin sheep

\begin{tabular}{|c|c|c|c|c|c|c|}
\hline Model & $s^{2} a$ & $s^{2} m$ & $\mathbf{s}_{\mathrm{am}}$ & $s^{2} p e$ & $s^{2} e$ & $s^{2} p$ \\
\hline \multicolumn{7}{|c|}{ Birth weight } \\
\hline M1 & 0.1281 & & & & 0.2711 & 0.3991 \\
\hline M2 & 0.0582 & & & 0.0474 & 0.2831 & 0.3886 \\
\hline M3 & 0.0525 & 0.0475 & & & 0.2886 & 0.3885 \\
\hline M4 & 0.0543 & 0.0199 & & 0.0290 & 0.2852 & 0.3883 \\
\hline M5 & 0.0713 & 0.0913 & -0.0487 & & 0.2773 & 0.3912 \\
\hline M6 & 0.0703 & 0.0649 & -0.0419 & 0.0212 & 0.2763 & 0.3907 \\
\hline \multicolumn{7}{|c|}{ Weaning weight at $75 \mathrm{~d}$} \\
\hline M1 & 3.9494 & & & & 6.9847 & 10.9340 \\
\hline M2 & 2.4317 & & & 0.9846 & 7.2803 & 10.6966 \\
\hline M3 & 4.0112 & 0.7457 & & & 6.1710 & 10.9279 \\
\hline M4 & 2.4318 & 0.0000 & & 0.9846 & 7.2803 & 10.6966 \\
\hline M5 & 1.9286 & 1.1868 & -0.2580 & & 7.4172 & 10.2745 \\
\hline M6 & 3.2277 & 0.8571 & -1.4087 & 1.2787 & 6.8511 & 10.8059 \\
\hline \multicolumn{7}{|c|}{ Adjusted weight at $120 \mathrm{~d}$} \\
\hline M1 & 4.3928 & & & & 6.3464 & 10.7392 \\
\hline M2 & 1.9304 & & & 1.2715 & 7.1301 & 10.3320 \\
\hline M3 & 1.8056 & 1.3079 & & & 7.2078 & 10.3212 \\
\hline M4 & 1.8143 & 0.8831 & & 0.4366 & 7.1862 & 10.3201 \\
\hline M5 & 2.1136 & 2.6051 & -1.3724 & & 7.0207 & 10.3671 \\
\hline M6 & 2.1124 & 2.5814 & -1.3646 & 0.0167 & 7.0209 & 10.8059 \\
\hline
\end{tabular}


vector de efectos genéticos aditivos directos aleatorios; $\mathbf{m}=$ vector de efectos genéticos maternos aleatorios; $\mathbf{c}=$ vector de efectos ambientales permanentes aleatorios; $\mathbf{e}=$ vector $\mathrm{de}$ efectos residuales aleatorios; $\mathbf{X}, \mathbf{Z}_{\mathbf{a}}, \mathbf{Z}_{\mathbf{m}}, \mathbf{Z}_{\mathbf{c}}=$ matrices de incidencia que relacionan las observaciones con los efectos fijos, genéticos directos, genéticos maternos $\mathrm{y}$ ambientales permanentes, respectivamente; $\mathbf{A}=$ matriz de relaciones; $\sigma_{\mathrm{am}}=$ covarianza entre efectos genéticos directos y maternos.

La estructura de (co)varianzas de los efectos aleatorios en el análisis se puede describir como sigue:

$\mathrm{V}(\mathbf{a})=\mathbf{A} \sigma_{\mathrm{a}}^{2} ; \mathrm{V}(\mathbf{m})=\mathbf{A} \sigma_{\mathrm{m}}^{2} ; \mathrm{V}(\mathbf{c})=\mathbf{I}_{\mathbf{d}} \sigma_{\mathrm{c}}^{2} ; \mathrm{V}(\mathbf{e})$ $=\mathbf{I}_{\mathbf{n}} \sigma^{2} ; \operatorname{Cov}(\mathbf{a}, \mathbf{m})=\mathbf{A} \sigma_{\mathrm{am}}$

Donde $\sigma_{\mathrm{a}}^{2}=$ varianza del efecto genético directo; $\sigma_{\mathrm{m}}^{2}=$ varianza del efecto genético materno; $\sigma_{\mathrm{am}}=$
The (co)variance structure of random effects in the analysis can be described as:

$\mathrm{V}(\mathbf{a})=\mathbf{A} \sigma_{\mathrm{a}}^{2} ; \mathrm{V}(\mathbf{m})=\mathbf{A} \sigma_{\mathrm{m}}^{2} ; \mathrm{V}(\mathbf{c})=\mathbf{I}_{\mathbf{d}} \sigma_{\mathrm{c}}^{2} ; \mathrm{V}(\mathbf{e})$ $=\mathbf{I}_{\mathbf{n}} \sigma^{2}{ }_{\mathrm{e}} ; \operatorname{Cov}(\mathbf{a}, \mathbf{m})=\mathbf{A} \sigma_{\mathrm{am}}$

Where $\sigma^{2}{ }_{\mathrm{a}}=$ direct additive genetic variance; $\sigma^{2}{ }_{\mathrm{m}}=$ maternal additive genetic variance; $\sigma_{\mathrm{am}}=$ directmaternal additive genetic covariance; $\sigma^{2}{ }_{c}=$ maternal permanent environmental variance; $\sigma^{2}=$ residual variance; $\mathbf{I}_{\mathbf{d}}=$ identity matrix of order equal to the number of dams; $\mathbf{I}_{\mathbf{n}}=$ identity matrix of order equal to the number of observations.

Direct $\left(\mathrm{h}_{\mathrm{d}}^{2}\right)$ and maternal heritability $\left(\mathrm{h}_{\mathrm{d}}^{2}\right)$, and proportion of phenotypic variance due to permanent environmental effects $\left(c^{2}\right)$ were estimated based on the following quotients:

Cuadro 3. Estimadores de parámetros genéticos para características de crecimiento de borregos Katahdin

Table 3. Estimates of genetic parameters for growth traits of Katahdin sheep

\begin{tabular}{|c|c|c|c|c|c|c|c|}
\hline Model & $h^{2} a$ & $h^{2} \mathrm{~m}$ & $r_{a m}$ & $c^{2}$ & & $e^{2}$ & $-2 \log L$ \\
\hline \multicolumn{8}{|c|}{ Birth weight (BW) } \\
\hline M1 & $0.32(0.03)$ & & & & 0.68 & $(0.03)$ & 1884.9539085934 \\
\hline M2 & $0.15(0.02)$ & & & $0.12(0.01)$ & 0.73 & $(0.02)$ & 1802.4687191141 \\
\hline M3 & $0.14(0.02)$ & $0.12(0.01)$ & & & 0.74 & $(0.02)$ & 1805.4179946928 \\
\hline M4 & $0.14(0.02)$ & $0.05(0.03)$ & & $0.07(0.03)$ & 0.73 & $(0.02)$ & 1800.0279452018 \\
\hline M5 & $0.18(0.03)$ & $0.23(0.03)$ & $-0.60(0.26)$ & & 0.71 & $(0.02)$ & 1794.4345125177 \\
\hline M6 & $0.18(0.03)$ & $0.17(0.05)$ & $-0.62(0.33)$ & $0.05(0.03)$ & 0.71 & $(0.02)$ & $1791.8842517234^{\star \star}$ \\
\hline \multicolumn{8}{|c|}{ Weaning weight at $75 \mathrm{~d}(\mathrm{WW})$} \\
\hline M1 & $0.36(0.03)$ & & & & 0.64 & $(0.03)$ & 37507. 701365154 \\
\hline M2 & $0.23(0.03)$ & & & $0.09(0.02)$ & 0.68 & $(0.02)$ & 37464.7986149105 \\
\hline M3 & $0.37(0.04)$ & $0.07(0.02)$ & & & 0.56 & $(0.03)$ & 37499.3681671549 \\
\hline M4 & $0.23(0.03)$ & $0.00(0.03)$ & & $0.09(0.03)$ & 0.68 & $(0.03)$ & 37464.7986147781 \\
\hline M5 & $0.19(0.03)$ & $0.12(0.03)$ & $-0.17(0.24)$ & & 0.72 & $(0.02)$ & 37480.8571575906 \\
\hline M6 & $0.30(0.04)$ & $0.08(0.05)$ & $-0.85(0.54)$ & $0.12(0.03)$ & 0.63 & $(0.03)$ & $37451.7274913214^{\star *}$ \\
\hline \multicolumn{8}{|c|}{ Adjusted weight at $120 \mathrm{~d}(\mathrm{AW})$} \\
\hline M1 & $0.41(0.04$ & & & & 0.59 & $(0.04)$ & 21963.9877643854 \\
\hline M2 & $0.19(0.04)$ & & & $0.12(0.02)$ & 0.69 & $(0.04)$ & 21935.4887674003 \\
\hline M3 & $0.17(0.04)$ & $0.13(0.02)$ & & & 0.70 & $(0.04)$ & 21934.3257635180 \\
\hline M4 & $0.18(0.04)$ & $0.09(0.07)$ & & $0.04(0.07)$ & 0.70 & $(0.04)$ & 21933.9120793495 \\
\hline M5 & $0.20(0.05)$ & $0.25(0.07)$ & $-0.58(0.43)$ & & 0.68 & $(0.03)$ & $21931.3137709806^{\star *}$ \\
\hline M6 & $0.20(0.05)$ & $0.25(0.11)$ & $-0.58(0.49)$ & $0.12(0.07)$ & 0.63 & $(0.04)$ & 21931.3132771112 \\
\hline
\end{tabular}

$h^{2}{ }_{a}=$ direct heritability; $h^{2} m=$ maternal heritability; $r_{a, m}=$ genetic correlation between direct and maternal effects; $c^{2}=$ maternal permanent environmental variance as a proportion of phenotypic variance. ${ }^{* *}$ most appropriate model. 
covarianza entre efectos genéticos aditivos directos y maternos; $\sigma^{2}=$ varianza ambiental permanente materna; $\sigma^{2}=$ varianza residual; $\mathbf{I}_{\mathbf{d}}=$ matriz identidad con dimensión igual al número de madres; $\mathbf{I}_{\mathbf{n}}=$ matriz identidad con dimensión igual al número de observaciones.

Las heredabilidades directa $\left(\mathrm{h}_{\mathrm{d}}^{2}\right)$ y materna $\left(\mathrm{h}_{\mathrm{d}}^{2}\right)$, y la proporción de la varianza fenotípica debida a los efectos ambientales permanentes $\left(\mathrm{c}^{2}\right)$ se estimaron con base en las ecuaciones siguientes:

$\mathrm{h}_{\mathrm{d}}^{2}=\sigma_{\mathrm{a}}^{2} / \sigma_{\mathrm{p}}^{2}, \quad \mathrm{~h}_{\mathrm{m}}^{2}=\sigma_{\mathrm{m}}^{2} / \sigma_{\mathrm{p}}^{2}, \quad \mathrm{c}^{2}=\sigma_{\mathrm{c}}^{2} / \sigma_{\mathrm{p}}^{2}$

Donde $\sigma^{2}$ p es la varianza fenotípica.

Los modelos diferentes en un efecto aleatorio se compararon usando la prueba de razón de verosimilitud (LRT) para cada característica ${ }^{(14)}$. Se consideró que un efecto tenía una influencia significativa cuando su adición resultó en una reducción significativa en el -2(logaritmo de la verosimilitud), en comparación con el modelo en el que no se incluyó. Cuando el -2(logaritmo de la verosimilitud) fue significativamente diferente $(P<0.05)$ entre modelos, el modelo con más parámetros se consideró como el más apropiado.

LRT $=(-2 \log \mathrm{L}$ para el modelo con $n$ parámetros $)$ - (-2LogL para el modelo con $\mathrm{n}+1$ parámetros), donde $-2 \log L=$ menos dos veces el logaritmo de la verosimilitud. $\mathrm{h}_{\mathrm{d}}^{2}=\sigma_{\mathrm{a}}^{2} / \sigma_{\mathrm{p}}^{2}, \mathrm{~h}^{2}{ }_{\mathrm{m}}=\sigma^{2}{ }_{\mathrm{m}} / \sigma_{\mathrm{p}}^{2}, \mathrm{c}^{2}=\sigma_{\mathrm{c}}^{2} / \sigma_{\mathrm{p}}^{2}$

where $\sigma_{p}^{2}$ is the phenotypic variance.

Models that differed in only one random effect were compared using the likelihood-ratio test (LRT) for each trait ${ }^{(14)}$. An effect was considered to have a significant influence when its addition caused a significant decrease in -2(logarithm of the likelihood), in comparison with the model in which it was ignored. When -2(logarithm of the likelihood) differed significantly $(P<0.05)$ between models, the model that had more parameters was selected as the most appropriate.

$\mathrm{LRT}=(-2 \mathrm{Log} \mathrm{L}$ for model with $\mathrm{n}$ parameters $)-(-2 \mathrm{Log} \mathrm{L}$ for model with $n+1$ parameters), where $-2 \log L=$ minus twice the logarithm of the likelihood.

\section{RESULTS AND DISCUSSION}

Estimates of (co)variance components for BW, WW and AW estimated with the six models are presented in Table 2. Heritabilities and -2(logarithm of the likelihood) values for each model are given in Table 3. Model comparisons between traits are presented in Table 4.

\section{Birth weight}

Estimates of additive genetic variance, and direct heritability for BW depended on the model used,

Cuadro 4. Comparación entre modelos que ignoran e incluyen efectos maternos

Table 4. Comparison between models that ignore or include maternal effects

\begin{tabular}{|c|c|c|c|c|}
\hline Model comparison & BW & WW & AW & Hypothesis \\
\hline Model 2 vs Model 1 & $160.798^{* *}$ & $85.866^{\star *}$ & $-28.498^{\star *}$ & $\sigma^{2} p e^{=0} 0$ \\
\hline Model 3 vs Model 1 & $133.550^{* *}$ & $62.373^{* *}$ & $-29.662^{* *}$ & $\sigma^{2} m=0$ \\
\hline Model 4 vs Model 2 & -0.067 & -0.000 & $-1.577^{\star *}$ & $\sigma_{m}^{2}=0$ \\
\hline Model 4 vs Model 3 & $-27.316^{\star \star}$ & $-23.493^{\star *}$ & $-0.414^{\star *}$ & $\sigma^{2} p e^{=} 0$ \\
\hline Model 5 vs Model 3 & $-17.682^{\star *}$ & $-27.432^{* \star}$ & $-3.012^{\star *}$ & $\sigma_{\mathrm{am}}=0$ \\
\hline Model 6 vs Model 4 & $-7.712^{\star *}$ & $-15.251^{\star *}$ & $-2.598^{* *}$ & $\sigma_{\mathrm{am}}=0$ \\
\hline Model 6 vs Model 5 & $-17.346^{\star *}$ & $-11.312^{\star *}$ & -0.000 & $\sigma^{2} p e^{2} 0$ \\
\hline
\end{tabular}

** Addition effect was significant $(P<0.05)$.

$B W=$ birth weight; $W W=$ weaning weight; $A W=120 \mathrm{~d}$ adjusted weight. 


\section{RESULTADOS Y DISCUSION}

Los estimadores de los componentes de (co)varianza para BW, WW y AW obtenidos con los seis modelos se presentan en el Cuadro 2. Las heredabilidades y los valores de -2(logaritmo de la verosimilitud) para cada modelo se muestran en el Cuadro 3. Las comparaciones de los modelos se presentan en el Cuadro 4.

\section{Peso al nacimiento}

Los estimadores de la varianza genética aditiva y de la heredabilidad directa para BW dependen del modelo usado, variando de 0.05 a 0.12 , y de 0.18 a 0.32 , respectivamente. La inclusión del efecto genético materno o del ambiente permanente materno en el modelo disminuyó significativamente los valores de -2(logaritmo de la verosimilitud) $(P<0.01)$ en comparación con el modelo más sencillo (Modelo 1). Con base en los valores del logaritmo de la verosimilitud, el Modelo 6 fue significativamente mejor $(P<0.01)$ que los otros modelos. El Modelo 1, que ignoró los efectos maternos, resultó en estimadores más altos de varianza genética aditiva y heredabilidad directa. Estos resultados coinciden con estudios en los que los componentes de (co)varianza fueron sobrestimados cuando los efectos maternos no fueron considerados en el modelo animal $(4,13,15)$. En los Modelos 2 y 3 la inclusión del efecto ambiental materno y del efecto genético aditivo materno, respectivamente, redujo el estimador de la varianza genética aditiva y de la heredabilidad directa. El agregar los efectos maternos al modelo (Modelo 4) no resultó significativo. Incluir la covarianza directa-materna en los Modelos 5 y 6 resultó en estimadores más altos de varianza genética aditiva y heredabilidad directa en comparación con los Modelos 3 y 4 en los que no se incluyó. Además, el Modelo 6 reduce el estimador de la varianza del error en comparación con los otros modelos. El estimador de la covarianza genética directa-materna fue negativo tanto en el Modelo 5 como en el Modelo 6 (-0.048 y -0.041) pero el logaritmo de las verosimilitudes entre ellos no fue pequeño. Además, la correlación genética ranging from 0.05 to 0.12 , and from 0.18 to 0.32 , respectively. Adding either genetic or permanent environmental maternal effect in the model decreased the -2(logarithm of the likelihood) values significantly $(P<0.01)$ in comparison with the simplest model (Model 1). On the basis of logarithm of the likelihood values, Model 6 was significantly better $(P<0.01)$ than the other models. Model 1 , which ignored maternal effects, resulted in higher estimates for additive genetic variance and direct heritability. This result coincides with reports where (co)variance components were overestimated when maternal effects were not included in the animal model $(4,13,15)$. In Models 2 and 3 the addition of maternal environmental effect and maternal additive genetic effect, respectively, reduced the estimates for additive genetic variance and direct heritability. The addition of maternal effects to the model (Model 4) was not significant. The addition of direct-maternal covariance in Models 5 and 6 increased estimates for additive genetic variance and direct heritability compared to Models 3 and 4 that ignored it. Also, Model 6 reduced the estimate of the error variance in comparison with the other models. The estimate of the directmaternal genetic covariance was negative for both, Model 5 and Model 6 (-0.048 and -0.041) but the logarithm of the likelihoods between them was not small. Also, direct maternal genetic correlation was negative for both models. Inclusion of the permanent environmental dam effect reduced the estimate of $\sigma^{2} \mathrm{~m}$ and $\mathrm{h}^{2} \mathrm{~m}$. When permanent environmental effects due to dam were not fitted (Models 3 and 4) these tended to increase the estimate of $\sigma^{2}$, inflating those values.

The most appropriate model (Model 6) for birth weight included both maternal genetic and permanent environmental effects and the directmaternal covariance. Snyman et al ${ }^{(16)}$ reported that a model which included both additive maternal and maternal permanent environmental effects, but not direct-maternal covariance, was the most appropriate model for birth weight of Afrino sheep. Ligda et al ${ }^{(11)}$ found that Model 6, which included both additive maternal and maternal permanent 
directa-materna fue negativa para ambos modelos. La inclusión de los efectos ambientales permanentes de la madre redujo el estimador de $\sigma^{2} \mathrm{~m} \mathrm{y} \mathrm{h}^{2} \mathrm{~m}$. Cuando los efectos ambientales permanentes de la madre no se incluyeron en el modelo (Modelos 3 y 4), el estimador de $\sigma^{2}$ e tendió a aumentar.

El Modelo 6 fue el más apropiado para peso al nacimiento e incluyó tanto el efecto genético y ambiental permanente materno, así como la covarianza directa-materna. Snyman et al(16) encontraron que un modelo que incluyó los efectos materno aditivo y del ambiente permanente, pero no la covarianza directa-materna, fue el más apropiado para peso al nacimiento en la raza Afrino. Ligda et al(11) encontraron que el Modelo 6, que incluye los efectos aditivo y ambiental permanente materno y la covarianza directa-materna, fue el mejor para peso al nacimiento de corderos Chios.

El estimador de la heredabilidad directa para peso al nacimiento ( 0.18 en el Modelo 6) fue similar al encontrado por Ligda et $a l^{(11)}$ y Ekiz et $a l^{(3)}$ en corderos Chios y Merino Turco. Sin embargo, otros autores (17-20) encontraron estimadores más altos de heredabilidad para peso al nacimiento en otras razas. En cambio, en otros trabajos $(5,21-24)$, se mencionan estimadores menores (Cuadro 5).

El estimador de heredabilidad materna para peso al nacimiento ( 0.17 con el Modelo 6) fue similar a los encontrados en la literatura $(11,18,22,24)$. El estimador del efecto ambiental permanente es similar a los encontrados por Snyman et al ${ }^{(16)}$ y Hossein-Zadeh y Ardalan(18). Sin embargo, los encontrados en Merino Turco(4) y en borregos Sabi(25) fueron mayores que los encontrados en este estudio.

\section{Peso al destete ajustado a 75 días}

Los estimadores de componentes de (co)varianza y de heredabilidades encontrados en la literatura se obtuvieron usando pesos ajustados a edades diferentes, por lo que el rango es muy alto y, por lo tanto, no son directamente comparables entre ellos. environmental effects and direct-maternal covariance, was the most suitable model for birth weight of Chios lambs.

The estimate of direct heritability of birth weight (0.18 in Model 6) was similar to the findings of Ligda et $a l^{(11)}$ and Ekiz et al(3) in Chios and Turkish Merino lambs. However, higher heritability estimates of birth weight were reported for other breeds $(17-20)$. Lower estimates were reported by others $(5,21-24)$, in several breeds (Table 5).

The maternal heritability estimate for birth weight ( 0.17 for Model 6) was in accordance with other findings $(11,18,22,24)$. The estimate of the permanent environmental effect was in agreement with the findings of Snyman et al(16) and Hossein-Zadeh and Ardalan(18). However, estimates for Turkish Merino $^{(4)}$ and for Sabi sheep ${ }^{(25)}$ were higher than estimates in our study.

\section{Weaning weight adjusted to $75 \mathrm{~d}$}

Estimates of (co)variance components and heritability found in the literature are from adjusted weights at different ages, then the range of estimates is very large, therefore those estimates are not directly comparable among them.

As with birth weight, highest estimates of direct additive genetic variance and heritability for weaning weight adjusted to $75 \mathrm{~d}$ were obtained with Model 1. The inclusion of maternal effects in the model led to a significant decrease in -2(logarithm of the likelihood) function. In this case, the effect of the permanent environment of the dam was significant, and the covariance between direct and maternal genetic effects was significant too, then Model 6 was considered as the most appropriate.

Maternal effects, genetic and environmental, followed the same trend as for birth weight, but were greater in magnitude. Estimates of additive genetic variance and maternal variance for WW (Table 3) ranged from 1.92 to $3.94 \mathrm{~kg}^{2}$ and from 0.74 to $1.18 \mathrm{~kg}^{2}$, among models. The estimates of additive genetic variance and maternal permanent 
Como sucedió en peso al nacimiento, los estimadores más altos de varianza genética aditiva y heredabilidad para peso al destete ajustado a 75 días se obtuvieron con el Modelo 1. La inclusión de los efectos maternos en el modelo llevó a una reducción significativa de la función -2(logaritmo de la verosimilitud). En este caso, el efecto del ambiente permanente materno fue significativo, así como la covarianza entre los efectos genéticos directo y materno, por lo que el Modelo 6 fue considerado como el más apropiado.

Los efectos maternos, genéticos y ambientales, mostraron la misma tendencia que para peso al nacimiento, pero fueron de mayor magnitud. Los estimadores de la varianza genética aditiva y materna para WW (Cuadro 3) variaron de 1.92 a $3.94 \mathrm{~kg}^{2}$ y de 0.74 a $1.18 \mathrm{~kg}^{2}$, dependiendo del modelo. Los estimadores de las varianzas genética aditiva y ambiental permanente materna fueron mayores que los encontrados en borregos Muzaffarnagari(15). El estimador de heredabilidad directa para WW fue mayor que el obtenido para BW, pero el estimador de heredabilidad materna fue menor, variando de 0.19 a 0.36 , y de 0.07 a 0.12 , respectivamente. Esto podría explicarse por el hecho de que es de esperar que los efectos maternos disminuyan en la medida en que crecen los corderos. El estimador de heredabilidad directa fue mayor que los valores encontrados en la literatura para otras razas, excepto por el obtenido por Gisaw et al ${ }^{(23)}$ para la raza Menz. Sin embargo, los estimadores de heredabilidad materna y de la varianza ambiental permanente fueron similares a los encontrados en la literatura para otras razas (Cuadro 5). En el Modelo 6, en el que se incluyeron los efectos genéticos y ambientales maternos, el $8 \%$ del total de la varianza se debió a los efectos genéticos maternos y el $12 \%$ a los efectos ambientales permanentes maternos. Los estimadores de la correlación genética entre los efectos directos y maternos variaron de -0.17 a -0.85 . Maniatis y Pollott ${ }^{(12)}$ consideraron que la correlación genética directa-materna era alta y negativa debido al reducido número de descendientes por hembra y a la poca información disponible por hembra. El environmental variance were higher than the findings in Muzaffarnagari sheep reported by Mandal et $a l(15)$. The estimate of direct heritability for WW was greater than that for BW, but the estimate of maternal heritability was smaller, ranging from 0.19 to 0.36 and from 0.07 to 0.12 , respectively. This could be probably explained by the fact that maternal effects are expected to decrease when lambs grow. The estimate of direct heritability was higher than the values for other breeds reported in the literature, except for the estimate reported by Gisaw et al(23) for the Menz breed. However, the estimates of maternal heritability and permanent environmental variance were according to the values reported in the literature for other breeds (Table 5). In model 6, in which both genetic and environmental maternal effects were taken into account, $8 \%$ of the total variance was attributed to maternal genetic effects and $12 \%$ to the maternal permanent environmental effect. The estimates for the genetic correlations between direct and maternal effects ranged from 0.17 to -0.85 . Maniatis and Pollott ${ }^{(12)}$ reported that the direct-maternal genetic correlation had a high negative value due to the small size of progeny group per dam and limited information from recorded dams. The main problem with field data sets is that often the types of covariance between relatives available in the data do not have sufficiently different expectations to allow the accurate partition of the phenotypic variance into all maternal (co)variance components.

\section{Postweaning weight adjusted to $120 \mathrm{~d}$}

Highest estimates of direct additive genetic variance and heritability for adjusted weight to $120 \mathrm{~d}$ were obtained with Model 1. The inclusion of maternal effects in the model led to a significant decrease in -2 (logarithm of the likelihood) function. In this trait, the maternal effect was significant, and the covariance between direct and maternal genetic effects was significant too, but the effect of the permanent environment of the dam was not significant, then Model 5 was considered as the most appropriate. 
Cuadro 5. Resumen de valores publicados de estudios previos de diferentes características de crecimiento en borregos

Table 5. Summary of published values from previous studies for different growth traits in sheep

\begin{tabular}{|c|c|c|c|c|c|c|c|c|c|c|c|}
\hline Breed & raits Weight & $s^{2} a$ & $\mathrm{~s}^{2} \mathrm{~m}$ & $s^{2} p e$ & $s^{2} p$ & $\mathrm{~s}_{\mathrm{am}}$ & $\mathrm{s}^{2} \mathrm{e}$ & $h^{2}$ & $h^{2} m$ & $\mathrm{r}_{\mathrm{am}}$ & Reference \\
\hline Turkish Merino & $\begin{array}{l}\text { Birth } \\
90 \mathrm{~d}\end{array}$ & $\begin{array}{l}0.11 \\
1.70\end{array}$ & - & $\begin{array}{l}0.12 \\
2-37\end{array}$ & $\begin{array}{c}0.63 \\
29.62\end{array}$ & - & $\begin{array}{c}0.38 \\
25.59\end{array}$ & $\begin{array}{l}0.18 \\
0.06\end{array}$ & - & - & Ekiz et al, 2004 \\
\hline Afrino & $\begin{array}{c}\text { Birth } \\
4-5 \mathrm{~m}\end{array}$ & $\begin{array}{l}0.09 \\
4.28\end{array}$ & $\begin{array}{l}0.04 \\
2.25\end{array}$ & $\begin{array}{c}0.05 \\
-\end{array}$ & $\begin{array}{c}0.41 \\
13.18\end{array}$ & - & $\begin{array}{l}0.23 \\
6.64\end{array}$ & $\begin{array}{l}0.22 \\
0.33\end{array}$ & $\begin{array}{l}0.09 \\
0.17\end{array}$ & $\begin{array}{c}0.12 \\
-\end{array}$ & Snyman et al, 1995 \\
\hline Chios & $\begin{array}{l}\text { Birth } \\
42 d\end{array}$ & $\begin{array}{l}0.09 \\
0.88\end{array}$ & $\begin{array}{l}0.10 \\
0.29\end{array}$ & $\begin{array}{l}0.08 \\
0.47\end{array}$ & $\begin{array}{l}0.51 \\
0.56\end{array}$ & $\overline{-0.04}$ & $\begin{array}{l}0.28 \\
3.99\end{array}$ & $\begin{array}{l}0.18 \\
0.16\end{array}$ & $\begin{array}{l}0.19 \\
0.05\end{array}$ & $\overline{-0.44}$ & Ligda et al, 2000 \\
\hline Kermani & $\begin{array}{l}\text { Birth } \\
3 \mathrm{~m}\end{array}$ & - & - & - & $\begin{array}{l}0.19 \\
9.68\end{array}$ & - & - & $\begin{array}{l}0.10 \\
0.22\end{array}$ & $\begin{array}{l}0.27 \\
0.19\end{array}$ & - & Bahreini Behzadi et al, 2007 \\
\hline Local breed & Birth & - & - & - & 0.59 & - & - & 0.42 & 0.33 & -0.60 & Al-Shorepy et al, 2001 \\
\hline Muzaffarnagari & $\begin{array}{l}\text { Birth } \\
15 d \\
30 d \\
45 d \\
60 d \\
75 d\end{array}$ & $\begin{array}{l}0.02 \\
0.02 \\
0.04 \\
1.01 \\
0.50 \\
2.34\end{array}$ & $\begin{array}{c}0.02 \\
0.06 \\
0.10 \\
- \\
- \\
-\end{array}$ & $\begin{array}{l}0.03 \\
0.17 \\
0.29 \\
0.40 \\
0.75 \\
0.66\end{array}$ & $\begin{array}{l}0.29 \\
1.10 \\
2.38 \\
3.75 \\
5.44 \\
8.05\end{array}$ & $\begin{array}{l}- \\
- \\
- \\
- \\
-\end{array}$ & $\begin{array}{l}0.22 \\
0.84 \\
1.95 \\
2.33 \\
4.19 \\
5.06\end{array}$ & $\begin{array}{l}0.08 \\
0.02 \\
0.02 \\
0.27 \\
0.09 \\
0.29\end{array}$ & $\begin{array}{c}0.08 \\
0.06 \\
0.04 \\
- \\
- \\
-\end{array}$ & $\begin{array}{l}- \\
- \\
- \\
- \\
-\end{array}$ & A. Mandal et al, 2006 \\
\hline Iranian Moghani & $\begin{array}{l}\text { Birth } \\
3 \mathrm{~m}\end{array}$ & - & - & $\begin{array}{l}0.05 \\
0.04\end{array}$ & - & $\frac{-}{-}$ & - & $\begin{array}{l}0.32 \\
0.21\end{array}$ & $\begin{array}{l}0.17 \\
0.09\end{array}$ & $-\overline{-0.23}$ & Hossein-Zadeh and Ardalan, 2010 \\
\hline Zandi & $\begin{array}{l}\text { Birth } \\
90 \mathrm{~d}\end{array}$ & $\begin{array}{l}- \\
-\end{array}$ & $\begin{array}{l}- \\
-\end{array}$ & 0.07 & $\begin{array}{l}- \\
-\end{array}$ & - & - & $\begin{array}{l}0.33 \\
0.08\end{array}$ & $\begin{array}{l}0.12 \\
0.02\end{array}$ & $\begin{array}{c}- \\
0.99\end{array}$ & $\begin{array}{l}\text { Ghafouri-Kesbi and } \\
\text { Eskandarinasab, } 2008\end{array}$ \\
\hline Purebreed & $\begin{array}{l}\text { Birth } \\
4 \mathrm{w}\end{array}$ & - & - & - & - & - & - & $\begin{array}{l}0.21 \\
0.21\end{array}$ & $\begin{array}{l}0.26 \\
0.18\end{array}$ & - & Szwaczkowski, et al, 2006 \\
\hline Katahdin & WA & 0.13 & - & 0.14 & 9.43 & - & - & 0.13 & - & 0.35 & Vanimisetti, et al, 2007 \\
\hline Dorper & $\begin{array}{l}\text { Birth } \\
45 d \\
100 d\end{array}$ & $\begin{array}{l}0.06 \\
1.55 \\
3.83\end{array}$ & $\begin{array}{l}0.05 \\
0.56 \\
1.83\end{array}$ & $\begin{array}{l}0.06 \\
0.61 \\
1.62\end{array}$ & $\begin{array}{c}0.53 \\
5.61 \\
19.32\end{array}$ & $\begin{array}{c}0.02 \\
-0.59 \\
-1.53\end{array}$ & $\begin{array}{c}0.34 \\
3.48 \\
13.58\end{array}$ & $\begin{array}{l}0.11 \\
0.28 \\
0.20\end{array}$ & $\begin{array}{l}0.10 \\
0.10 \\
0.10\end{array}$ & $\begin{array}{c}0.35 \\
-0.63 \\
-0.58\end{array}$ & Neser et al, 2001 \\
\hline Composite & $\begin{array}{l}\text { Birth } \\
7 \mathrm{w}\end{array}$ & - & - & $\begin{array}{l}0.09 \\
0.12\end{array}$ & $\begin{array}{c}1.02 \\
14.64\end{array}$ & - & - & $\begin{array}{l}0.09 \\
0.09\end{array}$ & $\begin{array}{l}0.17 \\
0.09\end{array}$ & $\begin{array}{r}0.01 \\
-0.39\end{array}$ & Mousa et al, 1999 \\
\hline Menz & $\begin{array}{l}\text { Birth } \\
3 \mathrm{~m}\end{array}$ & $\begin{array}{l}0.10 \\
1.91\end{array}$ & - & - & $\begin{array}{l}0.21 \\
4.01\end{array}$ & - & $\begin{array}{l}0.11 \\
2.10\end{array}$ & $\begin{array}{l}0.46 \\
0.48\end{array}$ & - & - & Gizaw et al, 2007 \\
\hline Beulah Specklefac & ced8 w & 1.96 & 0.96 & 1.37 & 7.43 & -0.99 & - & 0.23 & 0.11 & -0.73 & Husain et al, 2007 \\
\hline Mehraban & $\begin{array}{l}\text { Birth } \\
3 \mathrm{~m}\end{array}$ & $\begin{array}{l}0.06 \\
4.22\end{array}$ & $\begin{array}{l}0.04 \\
2.46\end{array}$ & - & $\begin{array}{c}0.21 \\
13.97\end{array}$ & - & $\begin{array}{l}0.11 \\
7.30\end{array}$ & $\begin{array}{l}0.30 \\
0.30\end{array}$ & $\begin{array}{l}0.17 \\
0.18\end{array}$ & - & Gamasaee et al, 2010 \\
\hline Suffolk & $\begin{array}{l}30 d \\
60 d \\
90 d\end{array}$ & $\begin{array}{l}- \\
-\end{array}$ & $\begin{array}{l}0.04 \\
0.05 \\
0.00\end{array}$ & $\begin{array}{l}0.19 \\
0.11 \\
0.19\end{array}$ & $\begin{array}{c}9.30 \\
29.9 \\
47.00\end{array}$ & $\begin{array}{l}- \\
-\end{array}$ & $\begin{array}{l}- \\
-\end{array}$ & $\begin{array}{c}0.16 \\
0.144 \\
0.21\end{array}$ & $\begin{array}{l}- \\
-\end{array}$ & $\begin{array}{l}- \\
- \\
-\end{array}$ & Notter, 1998 \\
\hline Polipay & $\begin{array}{l}30 d \\
60 d \\
90 d\end{array}$ & $\begin{array}{l}- \\
- \\
-\end{array}$ & $\begin{array}{l}0.17 \\
0.07 \\
0.00\end{array}$ & $\begin{array}{l}0.15 \\
0.15 \\
0.23\end{array}$ & $\begin{array}{c}6.30 \\
26.10 \\
29.90\end{array}$ & $\begin{array}{l}- \\
- \\
-\end{array}$ & $\begin{array}{l}- \\
- \\
-\end{array}$ & $\begin{array}{l}0.07 \\
0.08 \\
0.07\end{array}$ & $\begin{array}{l}- \\
-\end{array}$ & $\begin{array}{l}- \\
-\end{array}$ & Notter, 1998 \\
\hline Sabi & $\begin{array}{l}\text { Birth } \\
30 \mathrm{~d} \\
60 \mathrm{~d} \\
120 \mathrm{~d} \\
120 \mathrm{~d}\end{array}$ & $\begin{array}{l}- \\
- \\
- \\
8.87\end{array}$ & $\begin{array}{l}- \\
- \\
- \\
6.99\end{array}$ & $\begin{array}{l}0.08 \\
0.11 \\
0.06 \\
0.07 \\
3.89\end{array}$ & $\begin{array}{c}0.20 \\
2.14 \\
4.33 \\
9.11 \\
-\end{array}$ & $\begin{array}{l}- \\
- \\
- \\
4.39\end{array}$ & $\begin{array}{c}- \\
- \\
- \\
- \\
74.32\end{array}$ & $\begin{array}{l}0.25 \\
0.11 \\
0.13 \\
0.13 \\
0.09\end{array}$ & $\begin{array}{l}0.12 \\
0.03 \\
0.06 \\
0.06 \\
0.07\end{array}$ & $\begin{array}{l}- \\
- \\
- \\
0.55\end{array}$ & Matika et al, 2003 \\
\hline
\end{tabular}

$\mathrm{d}=$ days, $\mathrm{w}=$ week, $\mathrm{m}=$ month. $\mathrm{WA}=$ average weight of lambs weaned. See Tables 2 and 3 for abbreviations. 
problema principal con información de campo es que generalmente los tipos de covarianzas entre parientes disponibles en la información no tienen las suficientes esperanzas diferentes como para permitir la partición de la varianza fenotípica en todos los componentes de la (co)varianza materna.

\section{Peso posdestete ajustado a 120 días}

Los estimadores más altos de la varianza genética aditiva directa y de la heredabilidad para peso ajustado a 120 días se obtuvieron con el Modelo 1. La inclusión de los efectos maternos en el modelo resultó en una reducción significativa de la función -2(logaritmo de la verosimilitud). En esta característica, el efecto materno fue significativo, así como la covarianza entre efectos genéticos directos y maternos, pero el ambiente permanente de la madre no fue significativo, por lo que el Modelo 5 se consideró como el más apropiado.

Los componentes de covarianza y las heredabilidades siguieron la misma tendencia que en BW y WW pero tuvieron una mayor magnitud. Los estimadores de las varianzas genética aditiva y materna para AW variaron de 2.11 a $4.39 \mathrm{~kg}^{2}$ y de 0.88 a $2.60 \mathrm{~kg}^{2}$ dependiendo del modelo. Los estimadores de las varianzas genética aditiva y ambiental permanente materna con el modelo 5 fueron menores que las encontradas por Burfening y Kress ${ }^{(19)}$ en una raza compuesta. Los estimadores de heredabilidades directa y materna para AW variaron de 0.17 a 0.41 y de 0.09 a 0.25 , respectivamente. Con el Modelo 5, el estimador de la heredabilidad directa para AW fue mayor que el obtenido para BW pero menor que el de WW. El estimador de la heredabilidad directa para AW $(0.20)$ fue similar a los encontrados en la literatura científica(26-29). El estimador de la heredabilidad materna para AW (0.25) es similar a los obtenidos por van Wyk et al(30) y Cloete et al(28). Se han encontrado estimadores de heredabilidad directa menores en borregos Merino(30,31) y Targhee(32). Valores más altos se obtuvieron en borregos Afrino(31). En el Modelo 5, en el que se incluyeron los efectos maternos, el $25 \%$ de la varianza total fue explicada por los efectos genéticos maternos.
Covariance components and heritabilities followed the same trend as BW and WW but were greater in magnitude. Estimates of additive genetic variance and maternal variance for AW ranged from 2.11 to $4.39 \mathrm{~kg}^{2}$ and 0.88 to $2.60 \mathrm{~kg}^{2}$ among models. The estimates of additive genetic variance and maternal permanent environmental variance for model 5 were smaller than the findings in a Composite sheep reported by Burfening and Kress $(19)$. The estimates of direct and maternal heritability for AW ranged from 0.17 to 0.41 and 0.09 to 0.25 , respectively. For Model 5, the estimate of direct heritability for AW was greater than the one for BW but smaller than that for WW. The estimate of direct heritability for AW (0.20) was in accordance with those reported in the scientific literature (26-29). The estimate of maternal heritability for AW (0.25) was in agreement with the findings in the literature ${ }^{28,30)}$. Lower estimates of direct heritability were found in Merino sheep ${ }^{(31)}$, in $\operatorname{Dormer}^{(30)}$, and in Targhee ${ }^{(32)}$. Higher values were found in Afrino sheep reported by Snyman et al(31). In Model 5, in which maternal effects were taken into account, $25 \%$ of the total variance was attributed to maternal genetic effects. The estimates for the genetic correlations between direct and maternal effects were -0.58 for Models 5 and 6 .

\section{CONCLUSION AND IMPLICATIONS}

In conclusion, for $\mathrm{BW}$ and $\mathrm{WW}$ the most appropriate model was Model 6, which included direct and maternal effects, their covariance, and maternal permanent environmental effects. On the contrary, Model 5, which included direct and maternal genetic effects along with their covariance, was the most appropriate model for AW. Direct heritability for growth traits of Mexican Katahdin sheep could be overestimated if the animal model does not include maternal effects. Therefore, exclusion of maternal effects from the animal model resulted in an increase in the direct heritability for birth and weaning weights. Results from this study revealed that direct and maternal genetic effects are important for preweaning growth traits. Estimates of direct and maternal heritability obtained 
Los estimadores de las correlaciones genéticas entre efectos directos y maternos fueron -0.58 para los Modelos 5 y 6.

\section{CONCLUSIONES E IMPLICACIONES}

En conclusión, para BW y WW el modelo más apropiado fue el Modelo 6, que incluyó los efectos directo y materno, su covarianza, y el efecto ambiental permanente materno. Por otro lado, el Modelo 5, que incluyó los efectos genéticos directo y materno y su covarianza, fue el mejor modelo para AW. La heredabilidad directa para características de crecimiento de borregos Katahdin en México se sobreestima si el modelo animal utilizado no incluye los efectos maternos. Por lo tanto, la exclusión de los efectos maternos en el modelo animal resultó en una mayor heredabilidad directa en los pesos al nacimiento y destete. Los resultados de este estudio muestran que los efectos genéticos directos y maternos son importantes para las características de crecimiento predestete. Los estimadores de las heredabilidades directa y materna obtenidos indican que es posible obtener progreso en la selección para características de crecimiento pre y posdestete; por lo tanto, estas características pueden ser consideradas en programas de selección en esta raza(34). Sin embargo, en la selección de características de crecimiento debe tenerse en cuenta la alta correlación negativa que parece existir entre los efectos genéticos directos y maternos; las correlaciones negativas pueden ser el resultado de la estructura de la información, y no una relación negativa real(35). Por lo tanto es importante tener una alta proporción tanto de madres como de abuelas maternas con sus propios registros. La separación de los efectos genéticos maternos y ambientales permanentes maternos es posible sólo si se tienen registros repetidos de las hembras como madres. De esta manera, se necesitan varias generaciones bien conectadas y muchas relaciones entre parientes de las madres.

\section{LITERATURA CITADA}

1. Bradford GE. The role of maternal effects in animal breeding: VII. Maternal effects in sheep. J Anim Sci 1972;35:1324-1334. indicate that selection progress for pre and postweaning growth traits is feasible; therefore, these traits may be taken into consideration in any selection program on this breed(34). However, selection for growth traits would need careful consideration due to the strong and negative correlation that apparently exists between direct and maternal genetic effects; negative correlations could be statistical artifacts due to data structure, and not a real negative relationship ${ }^{(35)}$. Therefore a high proportion of both dams and maternal grand dams with their own records is essential. Partition of maternal genetic and maternal permanent environmental effects requires repeated records on individual mothers and the presence of these mothers in the data. Thus several well-linked generations of records and many relationships between relatives related to the mother would be needed.

End of english version

2. Wildeus S. Hair sheep genetic resources and their contribution to diversified small ruminant production in the United States. J Anim Sci 1997;75:630-640.

3. Ekiz B. Estimates of maternal effects for pre- and post-weaning daily gain in Turkish Merino Lambs. Turk J Vet Anim Sci 2005;29:399-407.

4. Ekiz B, Ozcan M, Yilmaz A. Estimates of genetic parameters for direct and maternal effects with different models on birth and weaning weights of Turkish Merino lambs. Turk J Vet Anim Sci 2004;28:383-389.

5. Bahreini Behzadi MR, Shahroudi FE, Van Vleck LD. Estimates of genetic parameters for growth traits in Kermani sheep. J Anim Breed Genet 2007;124:296-301.

6. Dixit SP, Dhillon JS, Singh G. Genetic and non-genetic parameter estimates for growth traits of Bharat Merino lambs. Small Ruminant Res 2001;42:101-104.

7. Husain SS, Wolf BT, Haresign W. Genetic parameters of lamb weights and ultrasonic muscle and fat depths in Beulah Specklefaced sheep. Small Rum Res 2007;70:116-123.

8. Safari E, Fogarty NM. Genetic parameters for Sheep production traits: Estimates from the literature. Technical Bulletin 49. NSW Agriculture, Orange NSW 2800, Australia. 2003.

9. Tosh JJ, Kemp KA. Estimation of Variance components for lamb weights in three sheep populations. J Anim Sci 1994;72:1184-1190.

10. Bromley CM, Snowder GD, Van Vleck LD. Genetic parameters among weight, prolificacy, and wool traits of Columbia, 
Polypay, Rambouillet, and Targhee sheep. J Anim Sci 2000;78:816-858.

11. Ligda Ch, Gabrilidis G, Papadopoulos Th, Georgoudis A. Investigation of direct and maternal genetic effects on birth and weaning weight of Chios lambs. Livest Prod Sci 2000;67:75-80.

12. Maniatis N, Pollott GE. The impact of data structure on genetic (co)variance components of early growth in sheep, estimated using an animal model with maternal effects. J Anim Sci 2003;81:101-108.

13. Abegaz S, van Wyk JB, Olivier JJ. Model comparison and genetic and environmental parameters estimates of growth and the Kleiber ratio in Horro sheep. S Afr J Anim Sci 2005;35:3040.

14. Meyer K. Variance components due to direct and maternal effects for growth traits of Australian beef cattle. Livest Prod Sci 1992;31-179.

15. Mandal A, Neser FWC, Rout PK, Roy R, Notter DR. Estimation of direct and maternal (co)variance components for pre-weaning growth traits in Muzzaffarnagari sheep. Livest Sci 2006;99:7989.

16. Snyman, MA, Erasmus GJ, van Wyk JB, Olivier JJ. Direct and maternal (co)variance components and heritability estimates for body weight at different ages and fleece traits in Afrino sheep. Livest Prod Sci 1995;44:229-235.

17. Al-Shorepy SA. Estimates of genetic parameters for direct and maternal effects on birth weight of local sheep in United Arab Emirates. Small Ruminant Res 2001;39:219-224.

18. Hossein-Zadeh NG, Ardalan M. Comparison of different models for the estimation of genetic parameters of body weight traits in Moghani sheep. Agric Food Sci 2010;19:207-213.

19. Burfening PJ, Kress DD. Direct and maternal effects on birth and weaning weight in sheep. Small Ruminant Res 1993;10:153-163.

20. Swackowski T, Wojtowski J, Stanislawska E, Gut A. Estimates of maternal genetic and permanent environmental effects in sheep. Arch Tierz Dummerstorf 2006;49:186-192.

21. Neser FWC, Erasmus GJ, van Wyk JB. Genetic parameter estimates for pre-weaning weight traits in Dorper sheep. Small Ruminant Res 2001;40:197-202.
22. Mousa E, Van Vleck LD, Leymaster KA. Genetic parameters for growth traits for a Composite Terminal sire breed of sheep. J Anim Sci 1999;77:1659-1665.

23. Gisaw S, Lemma S, Komen H, Van Arendock JAM. Estimates of genetic parameters and genetic trends for live weight and fleece traits in Menz sheep. Small Ruminant Res 2007;70: 145-153.

24. Gamasaee VA, Hafezian SH, Ahmadi A, Baneh H, Farhadi A, Mohamadi A. Estimation of genetic parameters for body weight at different ages in Mehraban sheep. Afr J Biotech 2010;9:52185223.

25. Matika O, van Wyk JB, Erasmus GJ, Baker RL. Genetic parameter estimates in Sabi sheep. Livest Prod Sci 2003;79:17-28.

26. Ghafouri-Kesbi F, Eskandarinasab MP. An evaluation of maternal influences on growth traits: the Zandi sheep breed of Iran as an example. J Anim Feed Sci 2008;17:519-529.

27. Gholizadeh M, Rahimi Mianji G, Hashemi M, Hafezian H. Genetic parameter estimates for birth and weaning weights in Raeini goats. Czech J Anim Sci 2010;55:30-36.

28. Cloete SWP, Greeff JC, Lewer RP. Environmental and genetic aspects of survival and early liveweight in Western Australian Merino sheep. S Afr J Anim Sci 2001;31:123-130.

29. Duguma G, Schoeman SJ, Cloete SWP, Jordaan GF. Genetic parameter estimates of early growth traits in the Tygerhoek Merino flock. S Afr J Anim Sci 2002;32:66-75.

30. van Wyk JB, Erasmus GJ, Konstantinov KV. Variance component and heritability estimates of early growth traits in the Elsenburg Dormer sheep stud. S Afr J Anim Sci 1993;23:72-76.

31. Snyman MA, Olivier JJ, Olivier WJ. Variance components and genetic parameters for body weight and fleece traits of Merino sheep in an arid environment. S Afr J Anim Sci 1996;26:1114.

32. Notter DR, Hough JD. Genetic parameter estimates for growth and fleece characteristics in Targhee sheep. J Anim Sci 1997;75:1729-1737.

33. Vanimisetti HB, Notter DR, Kuehn LA. Genetic (co)variance components for ewe productivity traits in Katahdin sheep. J Anim Sci 2007;85:60-68.

34. Hagger C. Litter, permanent environmental, ram-flock and genetic effects on early weight gain of lambs. J Anim Sci 1998;76:452-457. 